\title{
Dynamic contrast-enhanced MRI for response evaluation of non- small cell lung cancer in therapy with epidermal growth factor receptor tyrosine kinase inhibitors: a pilot study
}

\author{
Jae-Hun Kim ${ }^{1 \#}$, Ju Won Lee ${ }^{1,2 \#, ~ K e u n c h i l ~ P a r k ~}{ }^{3}$, Myung-Ju Ahn ${ }^{3}$,Jung Won Moon ${ }^{1,4}$, Soo-Youn Ham ${ }^{5}$, \\ Chin A Yi ${ }^{1}$
}

${ }^{1}$ Department of Radiology and the Center for Imaging Science, Samsung Medical Center, Sungkyunkwan University School of Medicine, Seoul, Korea; ${ }^{2}$ Department of Radiology, Human Medical Imaging and Intervention Center, Seoul, Korea; ${ }^{3}$ Division of Oncology, Department of Medicine, Samsung Medical Center, Sungkyunkwan University School of Medicine, Seoul, Korea; ${ }^{4}$ Department of Radiology, Hallym University Kangnam Sacred Heart Hospital, Seoul, Korea; ${ }^{5}$ Department of Radiology, Kangbuk Samsung Medical Center, Seoul, Korea

Contributions: (I) Conception and design: JH Kim, JW Lee, CA Yi; (II) Administrative support: K Park, MJ Ahn, SY Ham, JW Moon; (III) Provision of study materials or patients: JH Kim, JW Lee, CA Yi; (IV) Collection and assembly of data: JH Kim, JW Lee, K Park, CA Yi; (V) Data analysis and interpretation: JH Kim, JW Lee, JW Moon, CA Yi; (VI) Manuscript writing: All authors; (VII) Final approval of manuscript: All authors.

\#These authors contributed equally to this work as co-first authors.

Correspondence to: Chin A Yi, MD. Department of Radiology, Sungkyunkwan University School of Medicine, 81 Irwon-ro, Gangnam-gu, Seoul 06351, Korea. Email: cayi@skku.edu.

Backgroundk It is important to identify candidates who would benefit from target agent chemotherapy in advanced NSCLC patients. The purpose of this study is to evaluate the feasibility of DCE-MRI for early response evaluation in epidermal growth factor receptor (EGFR) tyrosine kinase inhibitors (TKIs) treatment in patients with NSCLC.

Methods: Seven patients were prospectively enrolled who have pathologically-proven NSCLC with EGFR mutations or at least 2 of the following factors; adenocarcinoma, female, or never-smokers. Patients were treated with gefitinib or erlotinib and the start of chemotherapy was denoted day 0. DCE-MRI was performed at day-1, day+7, and day+28. Longitudinal changes of perfusion parameters were quantified and compared to Response Evaluation Criteria in Solid Tumors (RECIST) results.

Results: Quantitative perfusion parameters; $K^{\text {trans }}, v_{e}$ and $v_{p}$ of the lung cancer showed a significant decrease at day+7 $(\mathrm{P}=0.016)$, but no further significant decrease between day+7 and day $+28(\mathrm{P}>0.05)$. Semiquantitative markers for tumor enhancement curve pattern; EA (enhancement amplitude), MS (maximum slope), and AUC (area under the curve) also showed a significant decrease at day+7 (P=0.016, 0.031, and 0.016 , respectively), but no further significant decrease between day +7 and day $+28(\mathrm{P}>0.05)$. When RECIST applied, all patient was in the stable disease at day 7 and three patients showed partial response $(\mathrm{PR})$ at day+28. All seven patients showed PR by the 3-month follow-up.

Conclusions: Perfusion parameters may be used as an early non-invasive imaging biomarker for the response evaluation of target agent treatment in NSCLC.

Keywords: Non-small cell lung cancer (NSCLC); perfusion magnetic resonance imaging (MRI); tumor angiogenesis

Submitted Dec 16, 2019. Accepted for publication Aug 10, 2020.

doi: 10.21037/apm-19-622

View this article at: http://dx.doi.org/10.21037/apm-19-622 


\section{Introduction}

Tumor angiogenesis is one of the most important biomarkers in cancers. Dynamic contrast-enhanced (DCE) magnetic resonance imaging (MRI) gives functional information on tumor angiogenesis and may reflect hemodynamic changes during treatment $(1,2)$. Several techniques have been tested to characterize tumor angiogenesis by analyzing signal intensity-time or attenuation-time curve with semi-quantitative parameters including peak enhancement, wash out, maximum slope and time-to-peak (3-5). However, these semi-quantitative parameters are limited to quantify the physiologic changes of tumor angiogenesis like increased permeability, lack of differentiation between venule and arteriole, and abundant extravascular space in cancer tissue. Relatively complicated pharmacokinetic modeling is needed to quantify these physiologic characteristics and begin their evaluation in oncologic practice (6).

Epidermal growth factor receptor tyrosine kinase inhibitors (EGFR-TKI), such as gefitinib, erlotinib, affatinib, osimerinitib, or dabominitib, are well-known target agents for the treatment of non-small cell lung cancer (NSCLC). Epidermal growth factor receptors (EGFR) are involved in tumor growth in many steps including cellular proliferation, angiogenesis, metastasis, and inhibition of apoptosis $(7,8)$. EGFR signaling upregulates the expression of multiple angiogenic factors and indirectly regulates angiogenesis by increasing the synthesis and release of proangiogenic factors from tumor cells (9). EGFR-TKI therapy has showed dramatic responses in subsets of NSCLC patients. Clinical trials have demonstrated better responses to EGFR-TKI in association with somatic mutations in EGFR (10-12) and with the following clinical characteristics: adenocarcinoma, no history of smoking, female sex, and Asian ethnicity (13-15). However, there remains a great deal of variation in predicting the responsiveness to EGFR-TKI therapy due to many factors, including primary or acquired resistance caused by additional mutations in the EGFR gene or KRAS mutation $(16,17)$. Therefore, non-invasive, repeatable, and early predictive parameters may benefit NSCLC patients by helping to determine a suitable treatment regimen, a practice revolutionized by the emergence of personalized therapy.

Although Response Evaluation Criteria in Solid Tumors (RECIST) is a well-established method for monitoring response in solid tumors, it cannot measure early changes in functional parameters, but only measure the changes of tumor size as parameters of response evaluation $(18,19)$. DCE-MRI is considered to measure tumor angiogenesis quantitatively with the perfusion parameters of a diffusible tracer, gadolinium chelates $(6,20)$, and may generate parameters which respond earlier than changes in tumor size. DCE-MRI has been tested as a novel biomarker in assessing anti-angiogenic effects in several kinds of tumors, including renal cell carcinoma, pancreatic cancer, and hepatic metastasis from colorectal cancer (21-23); however it has not been tested for the response evaluation of the lung cancer after EGFR-TKI therapy. The purpose of this study is to prospectively quantify the anti-angiogenic effect of EGFR-TKI at multiple time points using perfusion parameters from DCE-MRI in patients with lung cancer.

We present the following article in accordance with the MDAR reporting checklist (available at http://dx.doi. org/10.21037/apm-19-622).

\section{Methods}

The study was conducted in accordance with the Declaration of Helsinki (as revised in 2013). This study was approved by the institutional review board of Samsung Medical center (IRB file number: SMC 2009-07-066). All patients were given written informed consents.

\section{Patient selection}

Figure 1 is a diagram showing patient selection. Patients with pathologically-proven recurrent or metastatic NSCLC, at more than $3 \mathrm{~cm}$ along longest diameter, were assessed for EGFR-TKI therapy. The criteria for EGFR-TKI therapy were either the presence of somatic mutation of EGFR or more than 2 among the following requirements: (I) adenocarcinoma, (II) female sex, or (III) no history of smoking. Exclusion criteria included the following: abnormal renal function and contraindications for MRI (claustrophobia, cardiac pacemaker, etc.). Seven patients were recruited between September 2009 and March 2010 and they were treated with gefitinib (Iressa ${ }^{\circledR}$; AstraZeneca Pharmaceuticals, Wilmington, DE, USA) at a dose of $250 \mathrm{mg} /$ day orally on a 4 -week schedule or erlotinib (Tarceva $^{\circledR}$; Genentech, Inc., CA, USA or HoffmannLaRoche Ltd., Basel, Switzerland) at a dose of $150 \mathrm{mg} /$ day orally on 4-week schedule. All patients who agreed to 


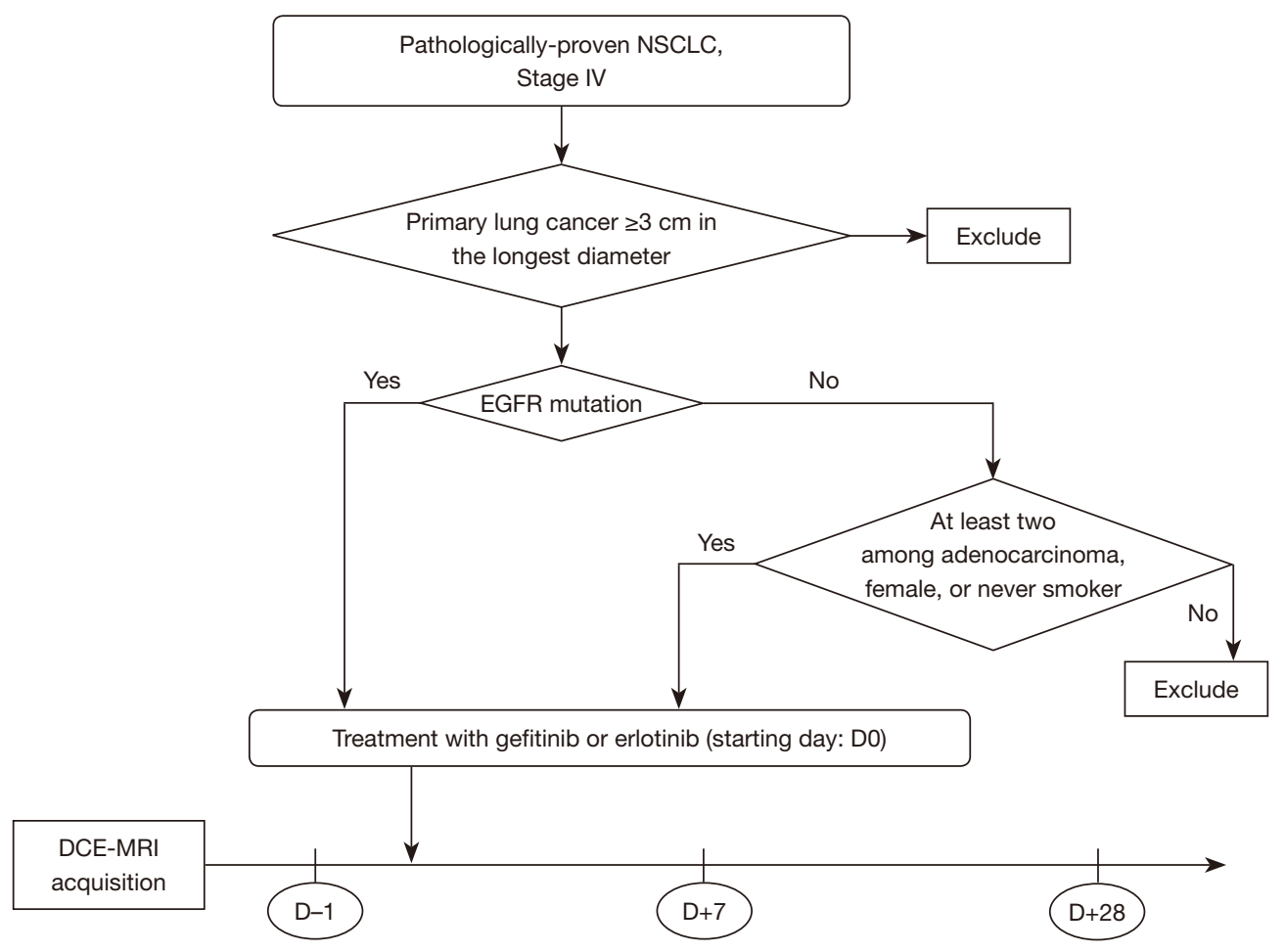

Figure 1 Flow diagram demonstrates patient selection algorithm.

participate in this study underwent a baseline DCE-MRI at day-1 (D-1). D0 denotes the starting date of chemotherapy. Follow-up DCE-MRI scans were scheduled at D+7 and $\mathrm{D}+28$. The window period is \pm 2 days.

\section{DCE-MRI acquisition}

DCE-MRI was performed with a 3-T MRI system (Achieva; Philips Medical Systems) equipped with a gradient system capable of ensuring maximum gradient amplitude of $80 \mathrm{mT} / \mathrm{m}$, a rise time of 0.2 milliseconds, and a slew rate of $200 \mathrm{~T} / \mathrm{m} / \mathrm{s}$. A sensitivity-encoding cardiac coil (SENSE, Philips Medical Systems) with a six-coil element was used for DCE-MRI covering the entire thorax.

The acquisition protocols are shown in Table 1. Transverse T2-weighted images with a multishot (MSH) spectral presaturation inversion recovery (SPIR) sequence was obtained for evaluation of the anatomy and the location of measurable disease. T1 mapping was done by multiple acquisitions using $\mathrm{T} 1$-weighted $3 \mathrm{D}$ spoiled gradientrecalled echo (SPGR) sequence of the thorax with different flip angles $\left(5^{\circ}, 15^{\circ}\right.$, and $\left.35^{\circ}\right)$. DCE-MRI of the lung parenchyma was optimized with the following acquisition parameters: TR, $5.6 \mathrm{~ms}$; TE, $1.47 \mathrm{~ms}$; flip angle, $35^{\circ}$; rectangular field of view, $350 \times 448 \mathrm{~mm}$; voxel size, $3 \times 3 \mathrm{~mm}$; reconstructed voxel size, $1.75 \times 1.74 \mathrm{~mm}$; reconstructed matrix, 256 $\times 256$; coronal orientation. The slab thickness was $100 \mathrm{~mm}$, and the slab was divided into 10 partitions to produce 10 images with $10 \mathrm{~mm}$ in section thickness. To increase acquisition speed, a sensitivity-encoding factor of 2 was applied in the phase-encoding direction. The temporal resolution was 3.0 seconds covering the whole thorax on coronal plane and a total of 60 dynamic scans were obtained. The entire DCE-MRI acquisition lasted up to 3 minutes imaging every 3 seconds. The patients were asked to breathe in as shallow a manner as possible during the MRI scanning. Gadolinium chelate (Dotarem; Guerbet, Aulnay-sous-Bois, France) was administered at a dose of $0.2 \mathrm{~mL}$ per kilogram of body weight by means of a power injector (Medrad, PA, USA) at a rate of $4 \mathrm{~mL} / \mathrm{s}$, followed by administration of $20 \mathrm{~mL}$ of normal saline in order to flush the tube.

All MR image data were directly interfaced to our PACS system (Pathspeed or Centricity 2.0, GE Healthcare), 
Table 1 Parameters for MR imaging sequences

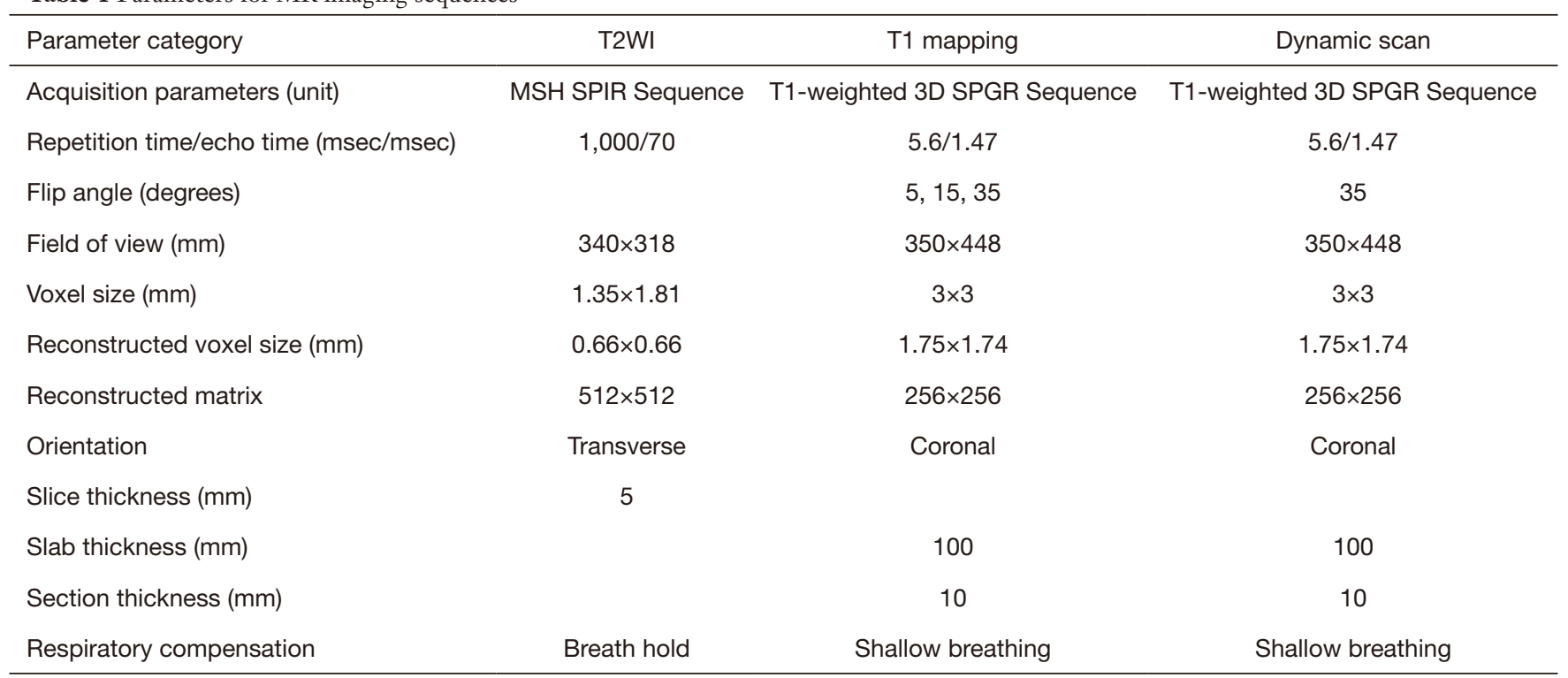

MSH, multishot; SPIR, spectral presaturation with inversion recovery; SPGR, spoiled gradient-recalled echo.

which displayed all image data on 4 monitors $(1,536 \times 2,048$ image matrices, 8 -bit viewable gray-scale, 60 -ft-lambert luminescence). Dynamic enhanced images were viewed on these monitors.

\section{Data analyses}

Concentration of contrast agent was estimated from MR signal intensity by computing the difference in longitudinal relaxation rate: $C(t)=\left(1 / T_{1}(t)-1 / T_{10}\right) / r_{1}$, where $T_{10}$ is pre-contrast $T_{1}$ value and $T_{1}(t)$ is post-contrast $T_{1}$ value, and $r_{1}$ denotes the longitudinal relaxivity $\left(4.5 \mathrm{~s}^{-1} \mathrm{mM}^{-1}\right.$ for blood) (24). The $T_{10}$ value of each voxel was computed using the variable flip angle method (25). The general equation for signal intensity values at a given flip angle is $S=S_{0}\left\{\sin \alpha\left[1-\exp \left(-T R / T_{10}\right)\right] /\left[1-\cos \alpha \exp \left(-T R / T_{10}\right)\right]\right\}$, where $T R$ is the repetition time, $\alpha$ is the flip angle, and $S_{0}$ is the equilibrium longitudinal magnetization. The $S_{0}$ and $T_{10}$ parameter were estimated by a linear least-squares method using three sets of SPGR sequences with different flip angles $\left(5^{\circ}, 15^{\circ}\right.$, and $\left.35^{\circ}\right)$. Following estimation of $T_{10}$ from the precontrast images, postcontrast $T_{1}(t)$ can be estimated as $1 / T_{1}(t)=-\ln \left\{1-\left(S_{\text {post }} / S_{\text {pre }}\right)\left[1-\exp \left(T R / T_{10}\right)\right]\right\} / T R$.

DCE-MRI for lung cancers was analyzed using semiquantitative and quantitative methods: the sigmoid fitting method for time-concentration curve pattern analysis and the pharmacokinetic modeling method, respectively.
For semi-quantitative analysis, a sigmoid equation was used (26):

$C(t)=A /\left[1+(B / t)^{C}\right]$, where $C(t)$ is the time-concentration curve averaged across all voxels within the region-ofinterests (ROIs) of the lung tumor, $A$ is the asymptotic enhancement amplitude (EA), and $B$ is time of half rising $\left(\mathrm{T}^{1 / 2} \mathrm{2}_{\text {max }}\right)$. Maximal slope (MS) was estimated as the first derivative of the above equation at $\mathrm{T}^{1 / 2} \mathrm{~m}_{\max }$ time point. Area under the curve (AUC) was calculated after sigmoid fitting of the time-concentration curve.

For quantitative analysis, the extended Kety twocompartment model was used (6): $C_{t}(t)=K^{\text {trans }} C_{p}(t) \otimes$ $\exp \left(-K^{\text {trans }} t / v_{e}\right)+v_{p} C_{p}(t)$, where $C_{t}$ is the concentration of contrast agent in the observed tissue, $C_{p}$ is the concentration in blood plasma, $v_{p}$ is the fractional blood plasma volume per unit volume of tissue, $K^{\text {trans }}$ is the volume transfer constant, and $v_{e}$ is the fractional extravascular extracellular space (EES) per unit volume of tissue. The $k_{\mathrm{ep}}$, flux rate constant between EES and blood plasma was calculated as $K^{\text {trans }} / v_{\text {e }}$.

The arterial input function (AIF) was determined semiautomatically by selecting an area within the pulmonary trunk or right ventricle among several candidates showing homogeneous Kendall's coefficients for time-concentration curves. The AIF at D-1 MRI was used as a baseline AIF and applied for analyzing data at D+7 and D+28 for each patient. 


\section{Response assessment}

The longest diameters of the measurable diseases were measured using an electronic caliper of the PACS system. The measurement of size was performed on the T2weighted axial images. The one-dimensional diameters were assessed by the RECIST criteria (27). If the sum of the diameters of target lesions showed a decrease of at least $30 \%$ on follow-up, it is considered as partial response (PR). Progressive disease was defined as an increase of at least $20 \%$ in the sum of diameters on follow-up. Stable disease was considered as having neither shrinkage sufficient to qualify for PR nor increase sufficient to qualify for PD on follow-up.

The longitudinal percentage change (\% change) in each perfusion parameter was evaluated at $\mathrm{D}+7$ and $\mathrm{D}+28$. Increases in $\mathrm{T}^{1 / 2}$ max $_{\max }$ and decreases in EA, MS, $K^{\text {trans }}, v_{\mathrm{e}}$ and $k_{\text {ep }}$, were considered anti-angiogenic effects.

\section{Statistical analysis}

For the analysis of changes in time-concentration curves after EGFR-TKIs therapy, the difference in EA, MS, T12/2 $\max$, and AUC between two time points was compared using the Wilcoxon signed rank test.

For the analysis of changes in perfusion parameters after EGFR-TKIs therapy, the differences in $K^{\text {trans }}, v_{\mathrm{e}}$, and $v_{\mathrm{p}}$ between two time points was compared using the Wilcoxon signed rank test.

A $\mathrm{P}$ value $<0.05$ indicated a statistical significance. Statistical analyses were performed using SAS (version 9.13, Cary, NC, USA).

\section{Results}

\section{Patients' characteristics}

All seven patients (four men and three women; median age, 53 years; age range, 46-67 years) underwent DCE-MRI before and after EGFR-TKIs therapy. The histological subtype of all patients was adenocarcinoma. Six out of the seven patients had EGFR mutations and the remaining one patient was a woman without a history of smoking. In terms of prior treatment: one patient had conventional chemotherapy and cyberknife surgery for brain metastasis; two patients had $1^{\text {st }}$ line chemotherapy; four patients had $1^{\text {st }}$ line plus another chemotherapy.

\section{Longitudinal changes in perfusion parameters}

The longitudinal changes in perfusion parameters (Figure 2) in the lung cancer were demonstrated with color changes on color map images. Lower scale colors were seen on perfusion map of the lung cancer on follow-up. Longitudinal parameter changes were demonstrated by changes in distribution of each voxel value of the whole lung cancer region. A bar graph of perfusion parameters depicts a shift toward the lower range of values on follow-up. Longitudinal changes of curve patterns were also quantitatively evaluated by sigmoid fitting of timeconcentration curve. These curves showed lower EA and maximum slope on follow-up, decreasing the AUC as a result.

Perfusion parameter and curve pattern analysis suggested anti-angiogenic effects after chemotherapy by showing a decrease in $K^{\text {trans }}, v_{\mathrm{e}}, v_{p}$, EA, MS, and AUC and an increase in $T^{1 / 2} 2_{\max }$ (Table 2). The distribution of $K^{\text {trans }}$ and $v_{\mathrm{e}}$ were significantly decreased at $\mathrm{D}+28$ after EGFR-TKI treatment on voxel based analysis $(\mathrm{P}=0.04$ and $<0.00$, respectively, Kolmogorov-Smirnov with Bonferroni's correction) (Figure 3). Concerning parameters from the curve pattern analysis, EA and MS were reduced and $\mathrm{T}^{1 / 2} \mathrm{~m}_{\max }$ was increased after the start of EGFR-TKI therapy, but the statistical significance of this finding was not proven (Table 2).

\section{Response assessment}

All seven patients who were clinically eligible for the EGFR-TKI treatment were responsive to EGFR-TKI treatment according to the traditional response evaluation method of RECIST in 3-month follow-up, but their responsiveness to EGFR-TKI treatment cannot be seen with RECIST in three patients on the 28th day of followup and none on the 7th day of follow-up. However, the perfusion parameters from DCE-MRI showed significant changes at an early date on the 7th day of follow-up in this study, being an early indication of responsiveness of EGFRTKI treatment.

\section{Discussion}

Changes in DCE-MRI parameters have been investigated to demonstrate efficacy of monoclonal antibodies targeting VEGF, TKIs and vascular-targeting agents for various kinds of tumors (28), but the lung cancers were rarely evaluated 
A

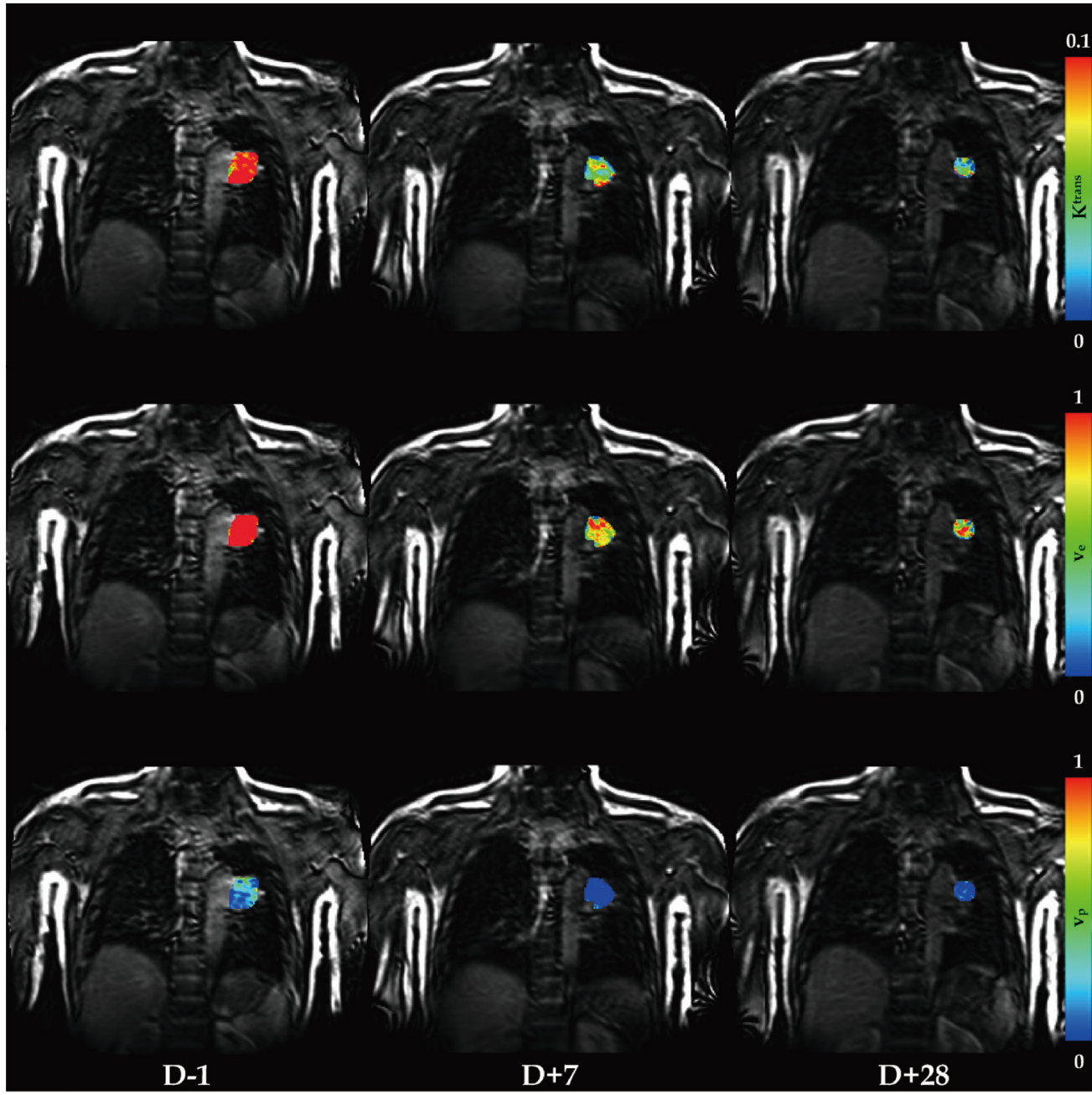

B

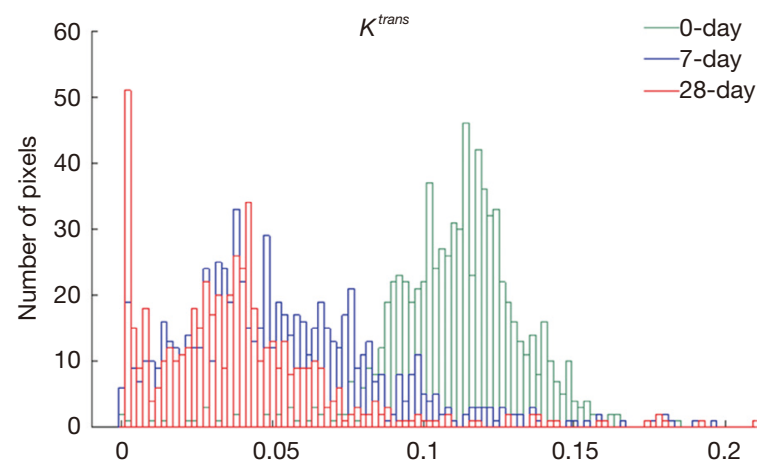

C

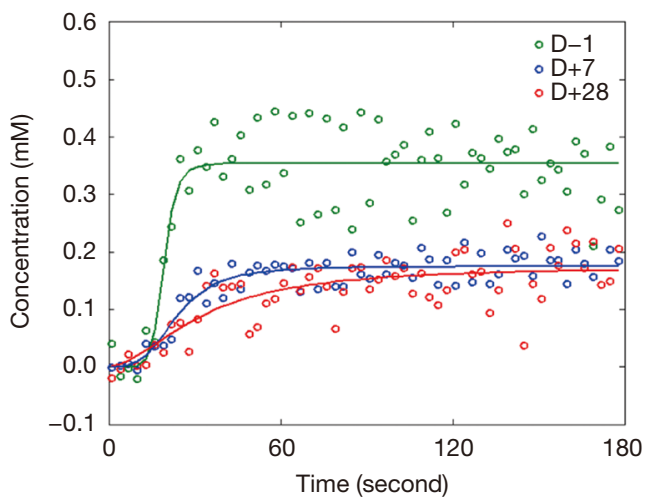

Figure 2 Longitudinal changes of perfusion parameters in a 46-year-old male patient with adenocarcinoma in the left upper lobe. The patient had an EGFR mutation and was treated with gefitinib. (A) Color maps visualize the decrease of $K^{\text {trans }}, \mathrm{v}_{\mathrm{e}}$ and $\mathrm{v}_{\mathrm{p}}$ in the lung cancer by showing color changes toward lower scale colors, especially striking changes between baseline and D+7. (B) Histogram demonstrates the voxel values of $K^{\text {trans }}$ of the whole tumor at each time point. The distribution of $K^{\text {trans }}$ shifts to the left, showing a decrease in $K^{\text {trans }}$ value after chemotherapy. (C) The pattern of time-concentration curves change after chemotherapy with interval decrease in EA and MS, especially between baseline and $\mathrm{D}+7$. 
Table 2 Longitudinal changes of perfusion parameters $(n=7)$

\begin{tabular}{|c|c|c|c|c|c|}
\hline \multirow{2}{*}{$\begin{array}{l}\text { Parameter } \\
\text { category }\end{array}$} & \multicolumn{3}{|c|}{ Mean } & \multicolumn{2}{|c|}{$P$ values* } \\
\hline & D-1 & $\mathrm{D}+7$ & $D+28$ & $\begin{array}{c}\mathrm{D}-1 \text { and } \\
\mathrm{D}+7\end{array}$ & $\begin{array}{c}D-7 \text { and } \\
D+28\end{array}$ \\
\hline \multicolumn{6}{|c|}{ Perfusion parameters } \\
\hline$K^{\text {trans }}$ & 0.096 & 0.038 & 0.042 & 0.016 & 0.375 \\
\hline$v_{e}$ & 0.724 & 0.399 & 0.389 & 0.016 & 0.813 \\
\hline$v_{p}$ & 0.190 & 0.031 & 0.069 & 0.016 & 0.469 \\
\hline \multicolumn{6}{|c|}{ Curve pattern analysis } \\
\hline EA & 0.322 & 0.144 & 0.144 & 0.016 & 1.000 \\
\hline MS & 0.043 & 0.010 & 0.020 & 0.031 & 0.578 \\
\hline $\mathrm{T} 1 / 2_{\max }$ & 8.576 & 9.188 & 9.340 & 0.688 & 0.813 \\
\hline AUC & 16.466 & 6.959 & 7.042 & 0.016 & 0.813 \\
\hline
\end{tabular}

*, Wilcoxon's signed rank test.

with DCE-MRI probably due to intrinsic difficulties to obtain enough signal from the air-filled organ and motion artifacts. There was only one published report about DCEMRI for the response evaluation of the lung cancer, which was treated with inhibitor of VEGF in combination with cytotoxic agent (29). However, to the best of our knowledge, there have been no studies regarding EGFR-TKIs as monotherapy. EGFR-TKIs are one of the most commonly used target agent for the NSCLCs, but their survival benefit outweighed only in subsets of NSCLC patients (30). Although clinical and molecular characteristics helped to screen NSCLC patients who can benefit from EGFR TKI treatment, there still remained a great deal of variation in predicting the responsiveness to EGFR-TKI therapy mainly due to primary or acquired resistance caused by additional mutations in the EGFR gene or KRAS mutation $(16,17)$. Therefore, non-invasive, repeatable, and early changing parameters are needed to guide EGFR-TKI treatment. DCE-MRI in our study visualized the longitudinal changes in perfusion parameters of the lung cancer on a voxel-byvoxel basis and quantified the early antivascular effects of EGFR-TKIs before these cytostatic agents make changes in volume of the tumor. We selected patients who were expected to show good response to EGFR-TKI therapy and all seven patients showed PR according to RECIST by the 3 months since the start of the chemotherapy. At the 7th day of follow-up after treatment, no patient met the criteria of PR without significant changes in tumor volume, but perfusion parameters indicated anti-angiogenic effect of chemotherapy by showing significant decrease in extent of perfusion parameters named $K^{\text {trans }}, v_{\mathrm{e}}$, and $v_{\mathrm{p}}$ and curve pattern parameters named EA, MS, and AUC.

Perfusion parameters of the whole lung cancer were calculated and displayed on a voxel-by-voxel basis and we could observe the heterogeneous characteristics of tumor angiogenesis in the lung cancer before and after the chemotherapy. In our analysis, longitudinal changes of $K^{\text {trans }}, v_{\mathrm{e}}$ and $v_{\mathrm{p}}$ were calculated for all voxel values within the tumor and the changes of distribution of pixels of lung cancer could be demonstrated on perfusion maps. This helped to understand the decreasing or increasing trend of perfusion parameters and how significant they were, although the spectrum of parameter values was wide. In the evaluation of treatment response of the brain tumors, these perfusion parametric maps could be correlated voxel-by-voxel before and after therapy and quantified treatment response on voxel basis by showing parametric response map (31). For the lung cancer, each perfusion parametric maps also could be generated before and after chemotherapy, but significant misregistration was expected in correlation of two longitudinal parametric maps.

Although EGFR-TKIs do not have direct antiangiogenic effect, they are known to have many indirect effects in diminishing angiogenesis. Our results demonstrated early and large percentage changes in perfusion parameters and in curve pattern parameters, which preceded changes in tumor size after EGFR-TKI treatment. This may suggest early anti-angiogenic effects of EGFR-TKIs decreasing permeability and EES volume in the lung cancer on treatment before shrinkage in the tumor volume. Perfusion parameters may be early and objective biomarkers for response evaluation in patients treated with anti-angiogenic drugs.

There are several limitations to our study. First, the number of patients was small since this was a feasibility study. Second, the correlation between perfusion parameters and long-term clinical outcome was not investigated. Third, patients who had tumors less than $3 \mathrm{~cm}$ were excluded from this study in order to measure a decrease in perfusion parameters and volume on follow-up image.

In conclusion, perfusion parameters from DCE-MRI can be quantified in patients with lung cancer. Quantitative biomarkers from DCE-MRI may play a role as objective parameters for tumor angiogenesis and early indicators for response evaluation of target agent treatment in NSCLC. 

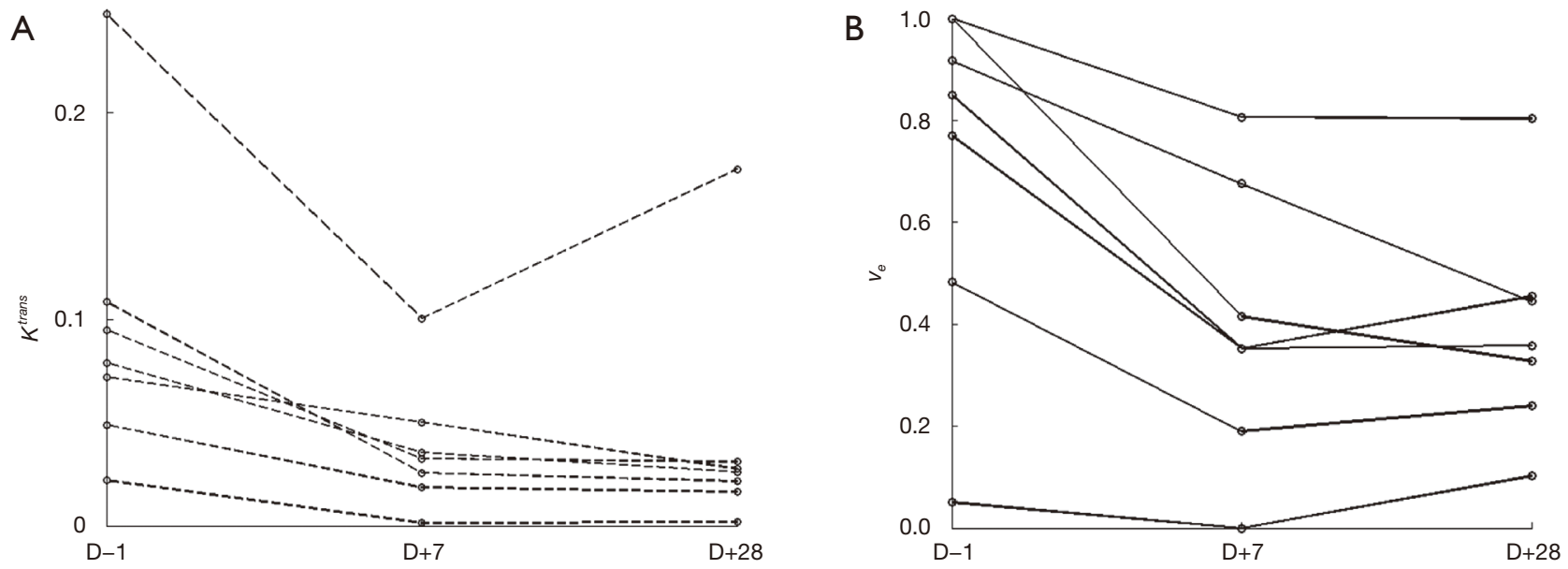

C

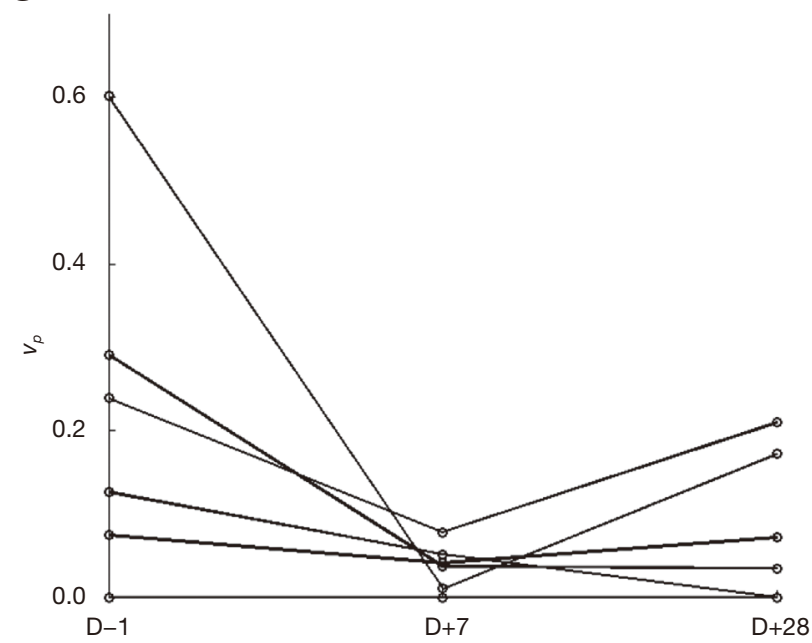

D

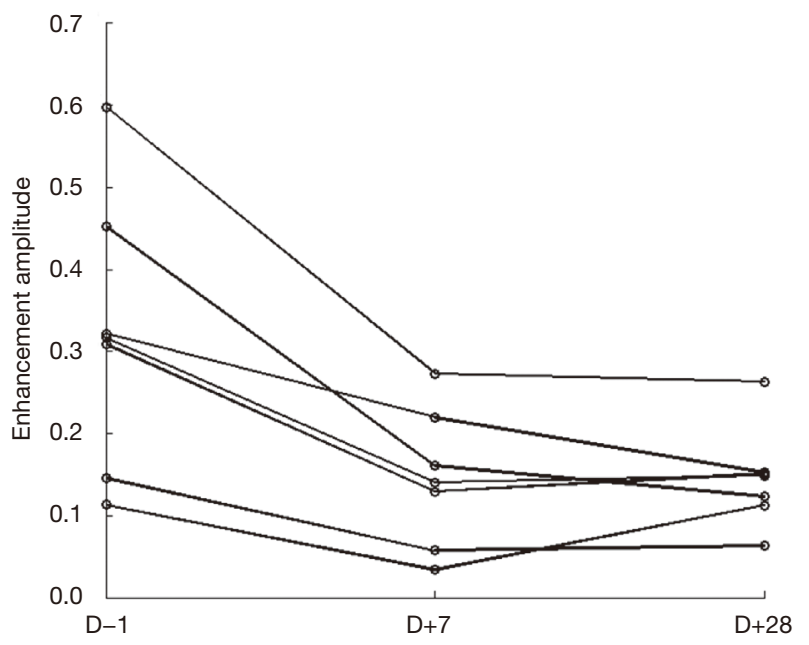

E

F
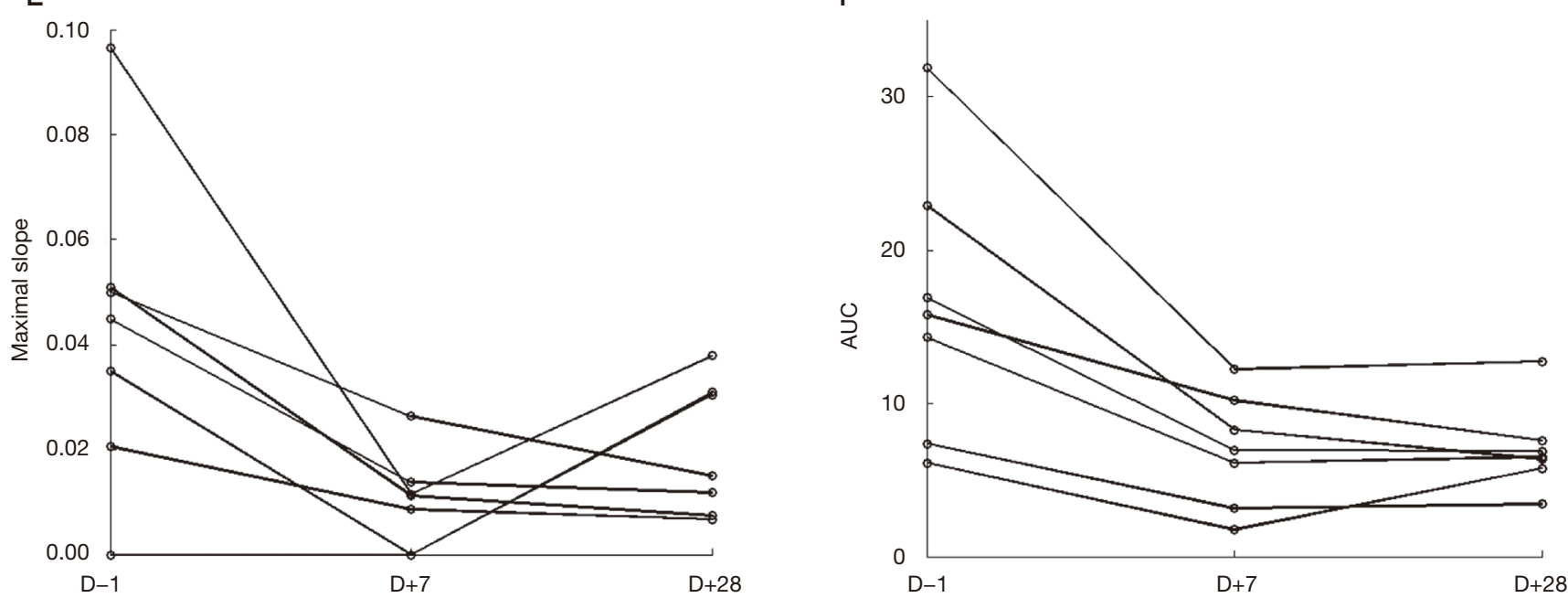

Figure 3 Changes in the following perfusion parameters in all seven patients: (A) $K^{\text {trans }},(\mathrm{B}) \mathrm{v}_{\mathrm{e}},(\mathrm{C}) \mathrm{v}_{\mathrm{p}}$, (D) EA, (E) MS, (F) AUC. Changes between $\mathrm{D}-1$ and $\mathrm{D}+7$ show significant drop for all these parameters. $(\mathrm{P}<0.05$ Wilcoxon's signed rank test). 


\section{Acknowledgments}

Funding: This work was supported by the National Research Foundation of Korea (NRF) grant funded by the Korea government (MEST) (NRF-2016R1A2B4013244 and NRF-2019R1A2C1011183).

\section{Footnote}

Reporting Checklist: The authors have completed the MDAR reporting checklist. Available at http://dx.doi.org/10.21037/ apm-19-622

Data Sharing Statement: Available at http://dx.doi. org/10.21037/apm-19-622

Conflicts of Interest: All authors have completed the ICMJE uniform disclosure form (available at http://dx.doi. org/10.21037/apm-19-622). The authors have no conflicts of interest to declare.

Ethical Statement: The authors are accountable for all aspects of the work in ensuring that questions related to the accuracy or integrity of any part of the work are appropriately investigated and resolved. The study was conducted in accordance with the Declaration of Helsinki (as revised in 2013). This study was approved by the institutional review board of Samsung Medical center (IRB file number: SMC 2009-07-066). All patients were given written informed consents. The study outcomes will not affect the future management of the patients.

Open Access Statement: This is an Open Access article distributed in accordance with the Creative Commons Attribution-NonCommercial-NoDerivs 4.0 International License (CC BY-NC-ND 4.0), which permits the noncommercial replication and distribution of the article with the strict proviso that no changes or edits are made and the original work is properly cited (including links to both the formal publication through the relevant DOI and the license). See: https://creativecommons.org/licenses/by-nc-nd/4.0/.

\section{References}

1. O'Connor JP, Jackson A, Parker GJ, et al. DCE-MRI biomarkers in the clinical evaluation of antiangiogenic and vascular disrupting agents. Br J Cancer 2007;96:189-95.

2. Zahra MA, Hollingsworth KG, Sala E, et al. Dynamic contrast-enhanced MRI as a predictor of tumour response to radiotherapy. Lancet Oncol 2007;8:63-74.

3. Yi CA, Lee KS, Kim EA, et al. Solitary pulmonary nodules: dynamic enhanced multi-detector row CT study and comparison with vascular endothelial growth factor and microvessel density. Radiology 2004;233:191-9.

4. Schaefer JF, Schneider V, Vollmar J, et al. Solitary pulmonary nodules: association between signal characteristics in dynamic contrast enhanced MRI and tumor angiogenesis. Lung Cancer 2006;53:39-49.

5. Jeong YJ, Lee KS, Jeong SY, et al. Solitary pulmonary nodule: characterization with combined wash-in and washout features at dynamic multi-detector row CT. Radiology 2005;237:675-83.

6. Tofts PS, Brix G, Buckley DL, et al. Estimating kinetic parameters from dynamic contrast-enhanced $\mathrm{T}(1)$ weighted MRI of a diffusable tracer: standardized quantities and symbols. J Magn Reson Imaging 1999;10:223-32.

7. Schlessinger J. Ligand-induced, receptor-mediated dimerization and activation of EGF receptor. Cell 2002;110:669-72.

8. Tsao MS, Sakurada A, Cutz JC, et al. Erlotinib in lung cancer - molecular and clinical predictors of outcome. $\mathrm{N}$ Engl J Med 2005;353:133-44.

9. Langer C, Soria JC. The role of anti-epidermal growth factor receptor and anti-vascular endothelial growth factor therapies in the treatment of non-small-cell lung cancer. Clin Lung Cancer 2010;11:82-90.

10. Bell DW, Lynch TJ, Haserlat SM, et al. Epidermal growth factor receptor mutations and gene amplification in nonsmall-cell lung cancer: molecular analysis of the IDEAL/ INTACT gefitinib trials. J Clin Oncol 2005;23:8081-92.

11. Eberhard DA, Johnson BE, Amler LC, et al. Mutations in the epidermal growth factor receptor and in KRAS are predictive and prognostic indicators in patients with non-small-cell lung cancer treated with chemotherapy alone and in combination with erlotinib. J Clin Oncol 2005;23:5900-9.

12. Reck M, Gatzemeier U. Gefitinib ("Iressa"): a new therapy for advanced non-small-cell lung cancer. Respir Med 2005;99:298-307.

13. Kris MG, Natale RB, Herbst RS, et al. Efficacy of gefitinib, an inhibitor of the epidermal growth factor receptor tyrosine kinase, in symptomatic patients with non-small cell lung cancer: a randomized trial. JAMA 2003;290:2149-58. 
14. Shepherd FA, Rodrigues Pereira J, Ciuleanu T, et al. Erlotinib in previously treated non-small-cell lung cancer. N Engl J Med 2005;353:123-32.

15. Thatcher N, Chang A, Parikh P, et al. Gefitinib plus best supportive care in previously treated patients with refractory advanced non-small-cell lung cancer: results from a randomised, placebo-controlled, multicentre study (Iressa Survival Evaluation in Lung Cancer). Lancet 2005;366:1527-37.

16. Hammerman PS, Janne PA, Johnson BE. Resistance to Epidermal Growth Factor Receptor Tyrosine Kinase Inhibitors in Non-Small Cell Lung Cancer. Clin Cancer Res 2009;15:7502-9.

17. De Luca A, Normanno N. Predictive biomarkers to tyrosine kinase inhibitors for the epidermal growth factor receptor in non-small-cell lung cancer. Curr Drug Targets 2010;11:851-64.

18. Suzuki C, Jacobsson H, Hatschek T, et al. Radiologic measurements of tumor response to treatment: practical approaches and limitations. Radiographics 2008;28:329-44.

19. Tuma RS. Sometimes size doesn't matter: reevaluating RECIST and tumor response rate endpoints. J Natl Cancer Inst 2006;98:1272-4.

20. Koh TS, Thng CH, Lee PS, et al. Hepatic metastases: in vivo assessment of perfusion parameters at dynamic contrast-enhanced MR imaging with dual-input two-compartment tracer kinetics model. Radiology 2008;249:307-20.

21. Hahn OM, Yang C, Medved M, et al. Dynamic contrastenhanced magnetic resonance imaging pharmacodynamic biomarker study of sorafenib in metastatic renal carcinoma. J Clin Oncol 2008;26:4572-8.

22. Akisik MF, Sandrasegaran K, Bu G, et al. Pancreatic cancer: utility of dynamic contrast-enhanced MR imaging in assessment of antiangiogenic therapy. Radiology 2010;256:441-9.

23. Vriens D, van Laarhoven HW, van Asten JJ, et al. Chemotherapy response monitoring of colorectal liver metastases by dynamic Gd-DTPA-enhanced MRI perfusion parameters and 18F-FDG PET metabolic rate. J Nucl Med 2009;50:1777-84.

24. Di Giovanni P, Azlan CA, Ahearn TS, et al. The accuracy of pharmacokinetic parameter measurement in DCE-MRI of the breast at 3 T. Phys Med Biol 2010;55:121-32.

25. Fram EK, Herfkens RJ, Johnson GA, et al. Rapid calculation of T1 using variable flip angle gradient refocused imaging. Magn Reson Imaging 1987;5:201-8.

26. Thomassin-Naggara I, Bazot M, Darai E, et al. Epithelial ovarian tumors: value of dynamic contrast-enhanced MR imaging and correlation with tumor angiogenesis. Radiology 2008;248:148-59.

27. Eisenhauer EA, Therasse P, Bogaerts J, et al. New response evaluation criteria in solid tumours: revised RECIST guideline (version 1.1). Eur J Cancer 2009;45:228-47.

28. O'Connor JP, Jackson A, Parker GJ, et al. Dynamic contrast-enhanced MRI in clinical trials of antivascular therapies. Nat Rev Clin Oncol 2012;9:167-77.

29. Chang YC, Yu CJ, Chen CM, et al. Dynamic contrastenhanced MRI in advanced nonsmall-cell lung cancer patients treated with first-line bevacizumab, gemcitabine, and cisplatin. J Magn Reson Imaging 2012;36:387-96.

30. Mok TS, Wu YL, Thongprasert S, et al. Gefitinib or carboplatin-paclitaxel in pulmonary adenocarcinoma. N Engl J Med 2009;361:947-57.

31. Tsien C, Galban CJ, Chenevert TL, et al. Parametric response map as an imaging biomarker to distinguish progression from pseudoprogression in high-grade glioma. J Clin Oncol 2010;28:2293-9.
Cite this article as: Kim JH, Lee JW, Park K, Ahn MJ, Moon JW, Ham SY, Yi CA. Dynamic contrast-enhanced MRI for response evaluation of non-small cell lung cancer in therapy with epidermal growth factor receptor tyrosine kinase inhibitors: a pilot study. Ann Palliat Med 2021;10(2):1589-1598. doi: 10.21037/apm-19-622 